\title{
Determination of the blocking temperature of magnetic nanoparticles: The good, the bad, and the ugly
}

\author{
I. J. Bruvera, ${ }^{1}$ P. Mendoza Zélis, ${ }^{1,2, a)}$ M. Pilar Calatayud, ${ }^{3,4}$ G. F. Goya,,${ }^{3,4}$ \\ and F. H. Sánchez ${ }^{1}$ \\ ${ }^{1}$ IFLP-CCT-La Plata-CONICET and Departamento de Física, Facultad de Ciencias Exactas, C. C. 67, \\ Universidad Nacional de La Plata, 1900 La Plata, Argentina \\ ${ }^{2}$ Departamento de Ciencias Básicas, Facultad de Ingeniería, Universidad Nacional de La Plata, \\ 1900 La Plata, Argentina \\ ${ }^{3}$ Aragon Institute of Nanoscience (INA), University of Zaragoza, 50018 Zaragoza, Spain \\ ${ }^{4}$ Condensed Matter Physics Department, Science Faculty, University of Zaragoza, 50009 Zaragoza, Spain
}

(Received 21 August 2015; accepted 29 October 2015; published online 13 November 2015)

\begin{abstract}
A numerically solved two-level Stoner-Wohlfarth model with thermal agitation is used to simulate Zero Field Cooling (ZFC)-Field Cooling (FC) curves of monosize and polysize samples and to determine the best method for obtaining a representative blocking temperature $T_{B}$ value of polysize samples. The results confirm a technique based on the $T$ derivative of the difference between $\mathrm{ZFC}$ and FC curves proposed by Micha et al. (the good) and demonstrate its relation with two alternative methods: the ZFC maximum (the bad) and the inflection point (the ugly). The derivative method is then applied to experimental data, obtaining the $T_{B}$ distribution of a polysize $\mathrm{Fe}_{3} \mathrm{O}_{4}$ nanoparticle sample suspended in hexane with an excellent agreement with TEM characterization. (C) 2015 AIP Publishing LLC. [http://dx.doi.org/10.1063/1.4935484]
\end{abstract}

\section{INTRODUCTION}

Magnetic nanoparticles (MNPs) are being extensively studied due to their multiple applications in technology ${ }^{1}$ and biomedicine. ${ }^{2-5}$ Particles with sizes in the range $[5,100] \mathrm{nm}$ (Ref. 6) present a magnetic behaviour determined by its volume, shape and composition, matrix viscosity, and temperature, among other factors. In the simplest (however very useful) model, MNPs of volume $V$ and saturation magnetization $M_{s}$ are considered as almost spherical ellipsoids with a permanent moment $m=M_{s} V$ and a preferential magnetization axis (easy axis) along which the anisotropy energy $E_{K}=$ $K V \sin ^{2}[\delta]$ is minimum, being $K$ the effective anisotropy density constant and $\delta$ the angle between $m$ and the easy axis. If the MNPs are fixed in the matrix and separated from each other by a distance $d>3 V^{1 / 3}$, dipolar interactions can be neglected ${ }^{7}$ and the energy of the system can be expressed as the sum of the anisotropy energy and the Zeeman energy $E_{H}=-m H \cos [\theta]$

$$
E=E_{K}+E_{H},
$$

with $\theta$ the angle between $m$ and $H$ (Fig. 1).

This configuration is usually called Stoner-Wohlfarth (SW) system in reference to these authors' publication of a work $^{8}$ in which they perform a numerical calculation of $M$ vs. $H$ curves of ordered systems with different orientations, i.e., systems of identical MNPs with a single value of $\phi$, and of the $M$ vs. $H$ curve of a disordered system, i.e., with a uniform distribution of $\phi$ values. Since no thermal agitation was considered by Stoner and Wohlfarth, their calculations were made just finding the positions $\theta_{i}$ of the minima of Equation (1) for each value of $H$.

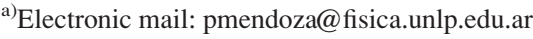

In order to calculate the temperature dependence of the magnetic response for MNPs systems, it is necessary to consider the effect of thermal fluctuations that allow transitions between stable configurations. Doing so, it is possible to simulate $M$ vs. $T$ experiments as the extensively performed Zero Field Cooling-Field Cooling (ZFC-FC) routine. In this kind of experiment, a sample is cooled from a temperature where all particles show superparamagnetic behaviour to the lowest reachable temperature (usually around $3 \mathrm{~K}$ ), then, a small constant field usually lower than $8 \mathrm{kA} / \mathrm{m}$ is applied, and the sample is heated to a temperature high enough to observe an initial growth and subsequent decrease of its magnetization, i.e., up to the temperature range where the sample shows

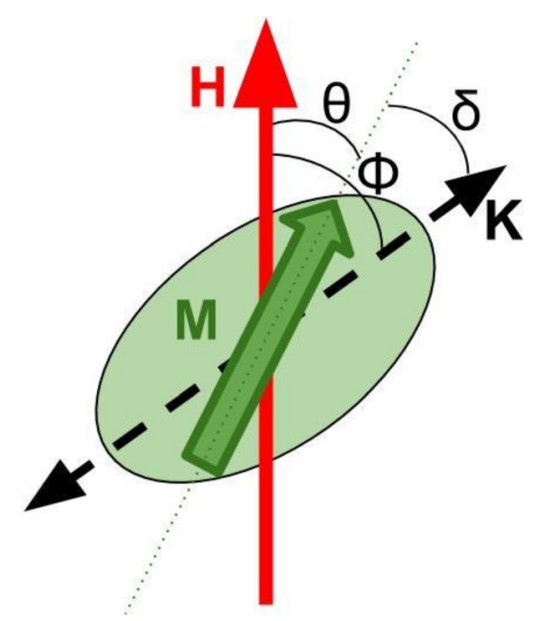

FIG. 1. MNP model. The energy is determined by the angle $\theta$ between the magnetization $M$ and the field $H$, and the angle $\delta$ between $M$ and the easy axis $K$. For calculation simplicity, the angle $\phi=\theta+\delta$ between $K$ and $H$ is used instead of $\delta$. 
again superparamagnetic behaviour. The sample is then cooled again to the lowest temperature with the constant field still applied.

In the ideal case of a monosize non interacting MNPs sample, a narrow temperature region should exist in which the system performs a transition between irreversible and reversible regimes. When heating under an applied field, the thermal energy $k T$ is initially much smaller than the anisotropy barrier $K V$ so the magnetization remains null. Due to the exponential dependence of the Néel relaxation time with temperature, ${ }^{9}$ when $k T \sim K V$, the magnetization grows rapidly up to its thermodynamic equilibrium value, defining the aforementioned transition region. The Blocking Temperature $T_{B}$ can be considered as the inflection point (IP) of this growing, and its experimental determination is an important goal of the MNPs characterization.

Real samples always present a size dispersion, usually reasonably well described by a log-normal distribution. Different particle size implies a different anisotropy barrier $K V$ and therefore a different $T_{B}$ for each size fraction, so in real ZFC-FC experiments, the blocking region is wide and a representative $T_{B}$ value of the ensemble is not well defined. There are several different criteria used to define a representative $T_{B}$ from ZFC-FC data of polysize samples. Some authors maintain the IP criterion, ${ }^{10}$ while others report the maximum ZFC magnetization temperature (MAX); ${ }^{11,12}$ both criteria are still under discussion. ${ }^{13-15}$ In an alternative approach, Micha et al. ${ }^{16}$ propose a method in which the $T_{B}$ distribution is obtained from the $\mathrm{T}$ derivative of the difference between ZFC and FC curves. An approximated theoretical justification for this method was presented by Mamiya et al. ${ }^{17}$

In this work, a SW model with thermal agitation is applied to obtain the temporal dependence of the magnetization $M(t)$ of an ordered system of identical MNPs in a similar way to the previous works of $\mathrm{Lu},{ }^{18}$ Usov, ${ }^{19}$ and Carrey. ${ }^{20}$ Temperature dependence $d M(T) / d T$ is then obtained in order to numerically simulate the ZFC-FC curves. In contrast to the method implemented by $\mathrm{Usov}^{21}$ where a stair-step approximation for the time evolution of the temperature was used, a continuous time evolution is considered. Finally, an ordered polysize system response is simulated by linear combination of the monosize curves weighted by a discrete lognormal distribution.

The validity of the method proposed by Micha et al. is verified by comparing the $T$ derivative of this ZFC-FC curve with the $T_{B}$ distribution obtained from the inflection points of each volume of the distribution. The resultant mean blocking temperature value $\left\langle T_{B}\right\rangle$ is then compared, for several volume distributions, with the commonly used criteria for a representative $T_{B}$ : the inflection point temperature IP and the maximum MAX of the ZFC curve.

Additionally, Micha's method is tested with experimental data of a frozen ferrofluid (FF) of magnetite MNPs suspended in hexane comparing the obtained $T_{B}$ distribution with the one calculated from the TEM size information. In order to obtain an ordered system, the ferrofluid is frozen while a large enough constant field is applied.

\section{MODEL}

A SW-like model with thermal agitation and zero width energy minima approximation was developed in order to obtain ZFC-FC curves of fixed MNPs with size dispersion. Only the simplest case of an ordered system was considered, with all the MNPs easy axes oriented in the direction of the field. This situation can be achieved experimentally by freezing a ferrofluid sample under a sufficiently strong applied field $(\sim 7 \mathrm{~T})$.

\section{A. Magnetization vs. time equation}

For a system of identical, fixed, non interacting MNPs of volume $V$, anisotropy constant $K$, and saturation magnetization $M_{s}$, with their anisotropy axes parallel to an external field $H$, the energy can be expressed as the sum of the anisotropy energy $E_{k}$ and the Zeeman energy $E_{h}{ }^{8}$

$$
\begin{aligned}
E=E_{k}+E_{h} & =K V \sin (\theta)^{2}-\mu_{0} M_{s} V \cos (\theta) \\
& =K V\left(\sin (\theta)^{2}-2 h \cos (\theta)\right),
\end{aligned}
$$

where $h=H / H_{k}$ and $H_{k}=\mu_{0} M_{s} / 2 \mathrm{~K}$.

In the range $\theta=[0,2 \pi]$, this energy landscape presents two minima of $E(0)=-2 K V h$ and $E(\pi)=2 K V h$ and a maximum of $E(\arccos (-h))=K V\left(1+h^{2}\right)$.

The frequency of thermal inversions between minima $i$ and $j$ is the inverse of the Nèel relaxation time $e^{22,23}$

$$
f_{i j}=f_{0} e^{-\Delta_{i j} /(k T)},
$$

with $f_{0}$ the "intrinsic frequency" times the Boltzmann "success probability" depending on the ratio between the thermal energy $k T$ and the barrier height $\Delta_{i j}$. The barrier between minima is symmetric for $h=0$ with $\Delta_{d u}=\Delta_{u d}=K V$ (naming $u$ and $d$ to $\theta=0$ and $\theta=\pi$ directions, respectively) and smaller for inversion to the field direction otherwise

$$
\begin{aligned}
& \Delta_{u d}=E(\arccos (-h))-E(\pi)=K V(1+h)^{2}, \\
& \Delta_{d u}=E(\arccos (-h))-E(0)=K V(1-h)^{2} .
\end{aligned}
$$

It is a good approximation to consider the same $f_{0}$ value for both frequencies. ${ }^{24}$

Sample magnetization $M$ in the direction of the applied field can be expressed in terms of saturation magnetization $M_{s}$ and the number of particles per unit volume magnetized in each direction $N_{u}$ and $N_{d}$

$$
M=\left(N_{u}-N_{d}\right) M_{s} / N=M_{s}\left(2 N_{u} / N-1\right),
$$

with $N$ the total number of particles per unit volume. So the time derivative of the magnetization can be written in terms of the population variation which is equal to the difference of the actual populations times the inversion probabilities

$$
\begin{gathered}
\frac{d M}{d t}=2 \frac{M_{s}}{N} \frac{d N_{u}}{d t}, \\
\frac{d N_{u}}{d t}=-\frac{d N_{d}}{d t}=f_{d \rightarrow u} N_{d}-f_{u \rightarrow d} N_{u},
\end{gathered}
$$


so the time derivative of the relative magnetization $\mathrm{m}$ is determined by the transcendental equation

$$
\frac{d m}{d t}=2 f_{0} e^{-C\left(1+h^{2}\right)}\{\sinh (2 C h)-m \cosh (2 C h)\},
$$

where $C=K V / k T$.

\section{B. Magnetization vs. temperature equation: ZFC-FC simulation}

Temperature dependence of the magnetization can be obtained from (8) via the equation

$$
\frac{d m}{d T}=\frac{d m}{d t} \frac{d t}{d T} .
$$

For a linear temperature variation $T(t)=B t+T_{0}$, the magnetization derivative is

$$
\frac{d m}{d T}=\frac{1}{B} \frac{d m}{d t}=\frac{2 f_{0}}{B} e^{-C\left(1+h^{2}\right)}\{\sinh (2 C h)-m \cosh (2 C h)\} .
$$

By solving this equation by means of numerical methods, it is possible to simulate a ZFC-FC experiment for a monosize sample. A Matlab script based on the ODE15s ${ }^{25}$ function was developed. An example of the result for a monosize assembly of ordered MNPs is shown in Figure 2. Line colours stand for different parts of the routine.

During the warming after zero field cooling (ZFCW for this chapter, usually called just ZFC), the exponential dependence of the inversion frequency with temperature in Equation (3) determines a narrow "blocking region" wherein the MNPs, which were "blocked" at low temperature, begin to respond to the field. Magnetization grows with temperature since the applied field has decreased the energy barrier for $\theta=\pi$ to $\theta=0$ inversion. The magnetization increasing reverts when thermal energy is much higher than the barrier, so the difference between inversion frequencies in each direction tends to disappear. The blocking temperature $T_{B}$ of

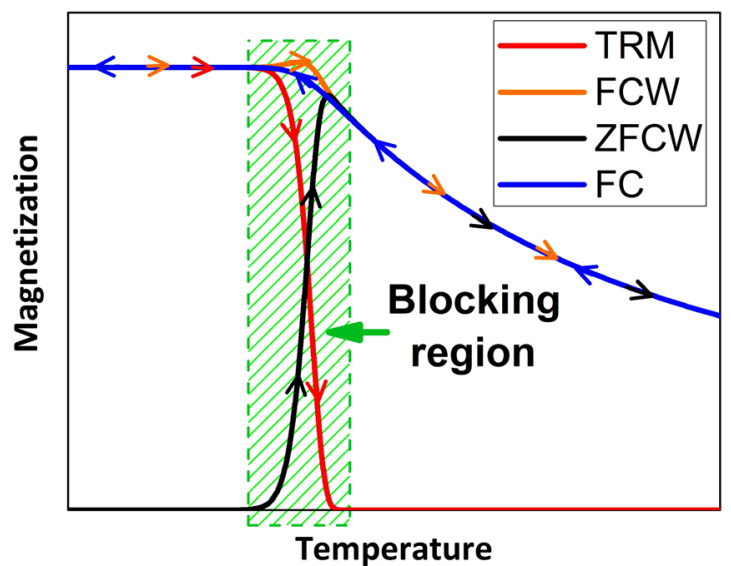

FIG. 2. Simulation result for an ordered assembly of MNPs. The system is first cooled with zero field applied from a high temperature where all particles show superparamagnetic behaviour (ZFC, no showed), then, the field is turned on and the system is heated beyond the blocking region (ZFCW). Maintaining the applied field, the system is cooled (FC). The final heating can be performed with (FCW) or without applied field (TRM). the system is then defined as the inflection point of the magnetization growing when heating.

When the system is cooled again (FC), magnetization grows monotonically while the barrier height difference between the two states becomes increasingly significant against thermal energy. This growth stops when thermal energy becomes too low for inversions to occur within the experimental window time. If the system is then heated maintaining the applied field (FCW), magnetization values are the same than FC except for the blocking region where there is a small increase due to the assembly getting closer to the equilibrium state. If the final warming is done with no applied field (Thermal Remanent Magnetism, TRM), magnetization drops to zero in the blocking region when thermal energy is enough for the wells populations to equilibrate.

The magnetization values $M_{p}$ for a polysize sample are obtained by linear addition of the $M_{V i}$ values for each contemplated size $V_{i}$, weighted by the corresponding volume and $\log$-normal distribution $\operatorname{LnN}\left(V_{i}\right)$ value

$$
M_{p}(T)=\frac{\sum_{i=1}^{N} M_{V_{i}}(T) V_{i} \operatorname{LnN}\left(V_{i}\right)}{\sum_{i=1}^{N} V_{i} \operatorname{LnN}\left(V_{i}\right)} .
$$

The $V_{i} \operatorname{LnN}\left(V_{i}\right)$ product stands for the relative volume distribution.

Figure 3 shows the comparison between ZFC-FC simulations for assemblies with different size dispersion expressed as the scale parameter $\sigma$ of the log-normal number distribution. A much wider transition region can be seen for the larger dispersion so the different aforementioned criteria would define very separated values for a representative $T_{B}$.

\section{BLOCKING TEMPERATURE DETERMINATION}

\section{A. Micha's method verification}

In order to verify Micha's method, ZFC-FC experiments were simulated for several polysize samples using different parameter sets varying $\sigma$ and the mean MNP radius. For each one of the used sets, the T derivative of the ZFC-FC difference was calculated. Then, the $T_{B}$ distribution was obtained

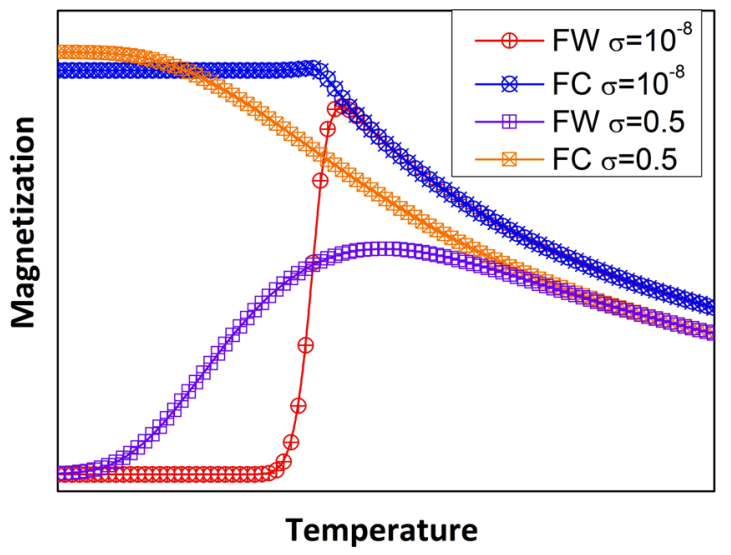

FIG. 3. Comparison between ZFC-FC simulations for assemblies with small dispersion $\left(\sigma=10^{-8}\right)$ and large dispersion $(\sigma=0.5)$. 


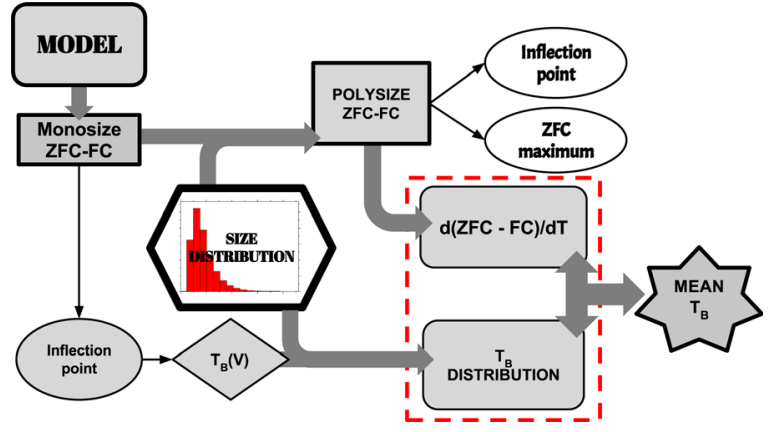

FIG. 4. Scheme of the method verification. Monosize ZFC-FC curves are simulated from the $\mathrm{dM}(\mathrm{T}) / \mathrm{dT}$ equations of the model. In one path, $T_{B}$ for every particle size is calculated as the IP of the monosize ZFC curve. In the other path, a polysize ZFC-FC curve is simulated by linear addition of the monosize values. Then, the $\mathrm{T}$ derivative of the difference $\mathrm{ZFC}-\mathrm{FC}$ is calculated.

from the monosize curves that were added to construct the polysize simulation in Equation (11): a ZFC curve was calculated for each class of the size distribution so each $T_{B}$ class comes from a volume class, maintaining the same relative height. Also, IP and MAX values of the polysize ZFC curve were calculated and compared with the mean value $\left\langle T_{B}\right\rangle$ of the distribution in each simulation (Fig. 4).

In all cases, the $T_{B}$ distribution and the ZFC-FC derivative are identical. Figure 5 shows the results for the simulation with $4.5 \mathrm{~nm}$ mean radius, $\sigma=0.5, K=16 \mathrm{~kJ} / \mathrm{m}^{3}$, and a $4 \mathrm{~K} / \mathrm{min}$ heating rate.

Also, for a set of ZFC-FC curves calculated with the same mean volume, saturation magnetization, heating rate, and anisotropy constant, by increasing scale parameter $\sigma$, $\left\langle T_{B}\right\rangle$ stays constant while the polysize curve IP shifts to smaller temperatures and MAX shifts in opposite direction. Figure 6 shows the results for $4.5 \mathrm{~nm}$ mean radius, $K=16 \mathrm{~kJ} /$ $\mathrm{m}^{3}, 4 \mathrm{~K} / \mathrm{min}$ heating rate, and $\sigma=[0.1,0.6]$.

This behaviour is the same in the whole studied size range. By normalizing IP values by the $T_{B}$ mean, all points fall in the same curve as shown in Figure 7, while the variation between MAX curves is small.

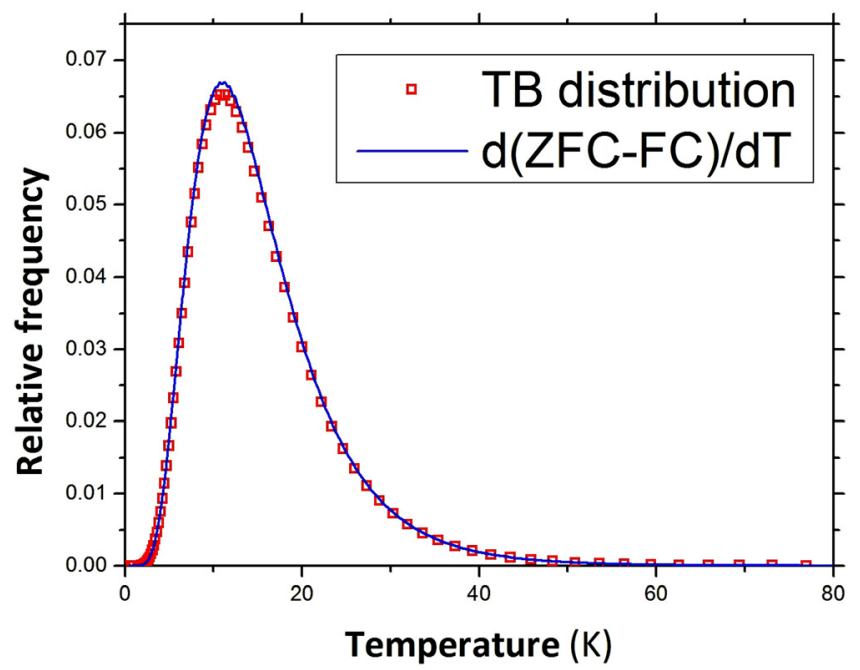

FIG. 5. Comparison between the $T_{B}$ distribution obtained directly from the size distribution used in the simulation and the derivative $d(\mathrm{ZFC}-\mathrm{FC}) / d T$ of the simulated curves.

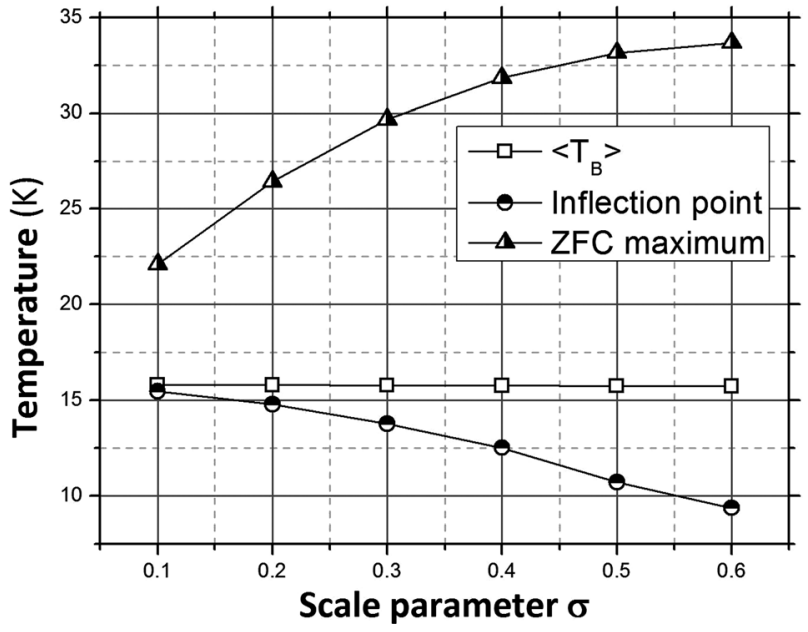

FIG. 6. Values of $T_{B}$ mean, MAX, and IP of the simulated curves as a function of the scale parameter $\sigma$ for $4.5 \mathrm{~nm}$ mean radius, $16 \mathrm{~kJ} / \mathrm{m}^{3}$ anisotropy constant, and $4 \mathrm{~K} / \mathrm{min}$ heating rate.

Varying the heating rate and $K$ does not affect the relation IP $/\left\langle T_{B}\right\rangle$. Meanwhile, the $\mathrm{MAX} /\left\langle T_{B}\right\rangle$ ratio changes strongly in the range $[0.04 ; 400] \mathrm{K} / \mathrm{min}$ and noticeably in the range $[1 ; 10] \mathrm{K} / \mathrm{min}$ and also depends on the $K$ value. Figure 8 shows the results of varying the heating rate for $R_{m}=10 \mathrm{~nm}, K=16 \mathrm{~kJ} / \mathrm{m}^{3}$, and $M_{s}=281 \mathrm{kA} / \mathrm{m}$. A parabolic fit was performed over the $I P /\left\langle T_{B}\right\rangle$ values obtained for all the simulations. The curve is universal with small fluctuations due to numeric resolution. The obtained polynomial with fitting errors is $\frac{I P}{\left\langle T_{B}\right\rangle}(\sigma)=1.00(2)-0.21(2) \sigma$ $-0.79(2) \sigma^{2}$.

\section{B. Experimental application}

Micha's analysis was conducted on ZFC-FC measurements of a FF of magnetite MNPs suspended in hexane with a concentration of 12(1)g/l. TEM images were taken in order to determine the size distribution of the particles (Fig. 9). A

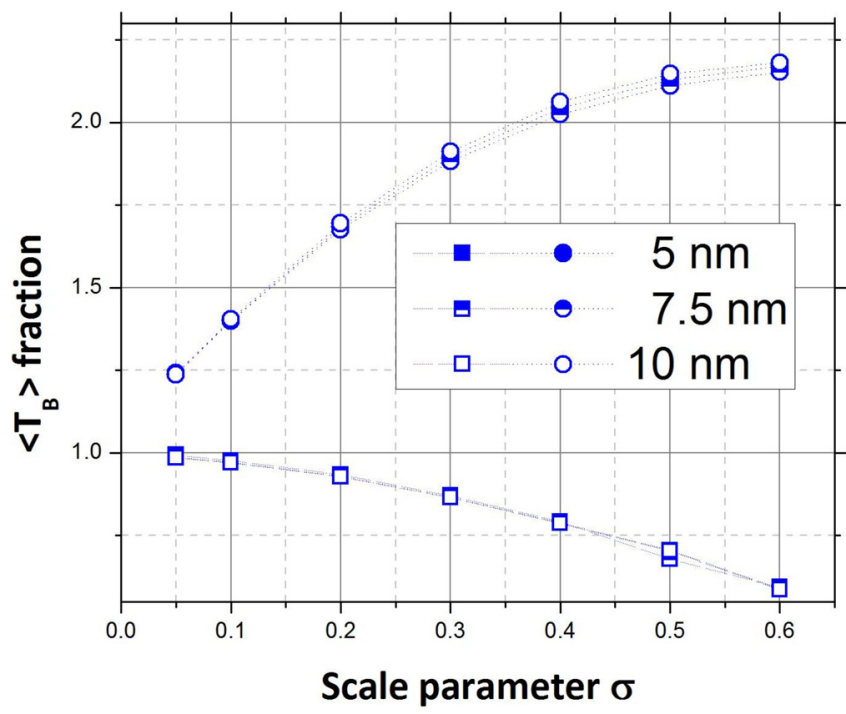

FIG. 7. Values of MAX (circles) and IP (squares) of the simulated curves divided by $T_{B}$ mean for different MNP radii. The behavior is the same for all sizes. 


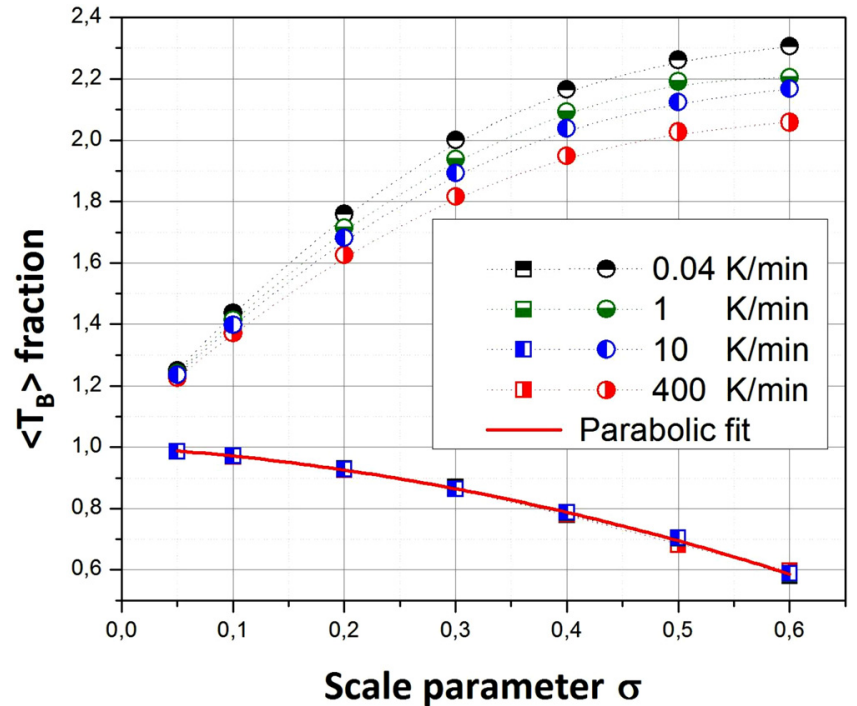

FIG. 8. MAX (circles) and IP (squares) relative to $\left\langle T_{B}\right\rangle$ for $10 \mathrm{~nm}$ NPM mean radius at different temperature rates. The $I P /\left\langle T_{B}\right\rangle$ values describe a universal parabolic curve.

narrow log-normal number diameter distribution $(\operatorname{LnN}(x))$ was obtained with a $9.5 \mathrm{~nm}$ mean and a $1.7 \mathrm{~nm}$ standard deviation. The relative TEM volume distribution was obtained from this results and fitted with a $x \operatorname{LnN}(x)$ function obtaining a scale parameter $\sigma=0.55(2)$.

The $\mathrm{ZFC}-\mathrm{FC}$ routine was carried out at a $2.4 \mathrm{~K} / \mathrm{min}$ rate and a $8 \mathrm{kA} / \mathrm{m}$ field on an encapsulated FF sample frozen under a $7 T$ field in order to obtain an ordered system with all MNP easy axes oriented parallel to the field. The ZFC-FC derivative was calculated and fitted with a $x \operatorname{LnN}(x)$ distribution using the TEM $\sigma$ as a fixed parameter with a very good correspondence (Figure 10).

Figure 11 shows the comparison between the $T_{B}$ distribution obtained from TEM information and the ZFC-FC derivative curve. The translation from TEM volume to $T_{B}$ was made considering the blocking condition in which the

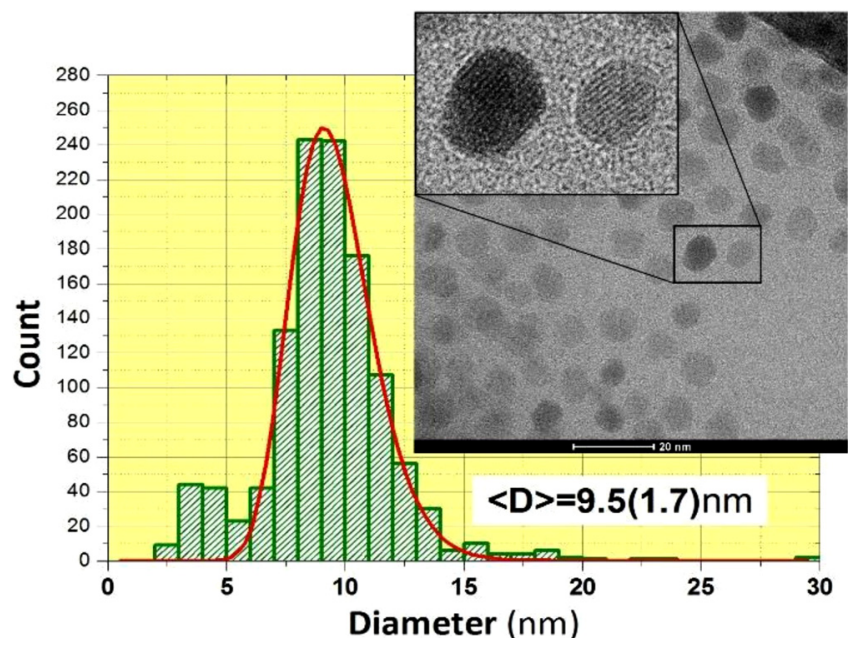

FIG. 9. Size distribution from TEM images. Inset: TEM image example with a magnification showing the crystallinity of the particles. The fitting left out the smallest MNP with small incidence in volumetric magnetic response.

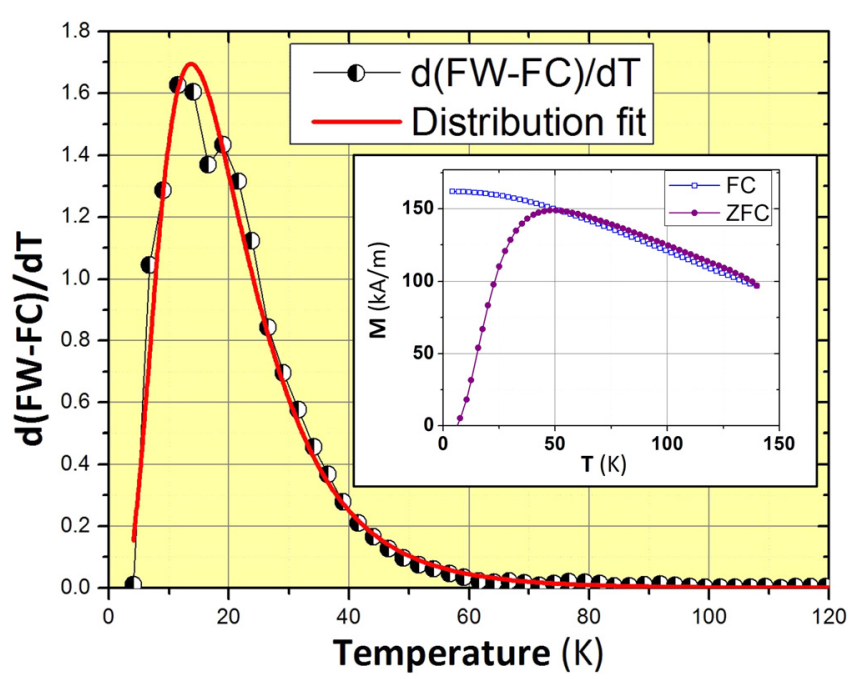

FIG. 10. Log-normal fit of the $d(\mathrm{ZFC}-\mathrm{FC}) / d T$ derivative. Inset: ZFC and FC experimental curves.

inversion time $\tau_{m}$ of the MNPs is approximately equal to the measurement time of the magnetization value

$$
\begin{aligned}
\tau(K, V, h, T) & =\tau_{0} \exp \left(\frac{K V}{k T_{B}}(1-h)^{2}\right) \approx \tau_{m} \\
& \Rightarrow T_{B}=\frac{K V(1-h)^{2}}{k \log \left(\tau_{m} / \tau_{0}\right)},
\end{aligned}
$$

where $\tau_{0}=1 / f_{0}$ is the inverse of the intrinsic inversion frequency. For a known volume distribution, this comparison can be used to determine the effective $K$ value as the one that maximizes the coincidence between TEM and ZFC-FC distributions. In this case, a value of $34(2) \mathrm{kJ} / \mathrm{m}^{3}$ was obtained with a very good correspondence between TEM and ZFC-FC data. This calculation implies some approximations: $M_{s}$ is considered independent from the temperature in the region of interest, and the relaxation time expression used for the blocking condition (Equation (12)) considers only the inversions in the direction of the field. While the first approximation is very reasonable, the blocking condition expression is accurate

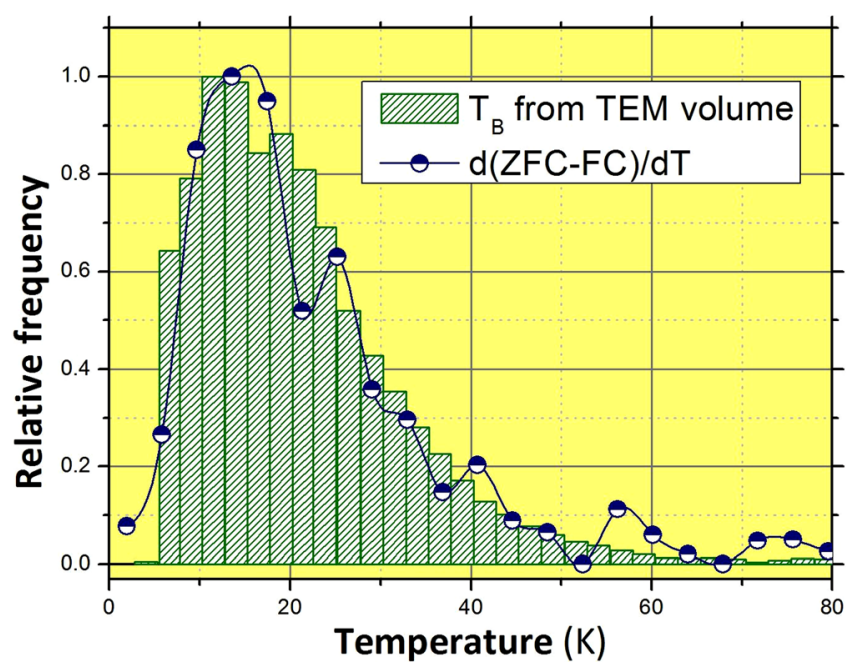

FIG. 11. $d(\mathrm{ZFC}-\mathrm{FC}) / d T$ derivative together with $T_{B}$ distribution from TEM volume obtained by fitting $K$ for maximum coincidence. 
only in experiments with high $\mu H /(k T)$ ratios, where the reversal frequencies are much smaller for the inversions to the antiparallel state, as it happens in the present case.

Additionally, the IP $/\left\langle T_{B}\right\rangle$ ratio was calculated obtaining a value of 0.7(1), compatible with polynomial expression obtained from the simulations.

\section{DISCUSSION AND CONCLUSIONS}

The validity of Micha's method to determine the $T_{B}$ distribution of non interacting MNPs assembly was demonstrated by numerical simulations and experimental data analysis.

A Stoner-Wolfarth model with thermal agitation was developed in order to simulate the ZFC-FC curves of polysize MNPs assemblies. From this simulation, it was clearly demonstrated that the temperature derivative of the ZFC-FC difference is in full coincidence with the $T_{B}$ distribution of the sample, calculated as the inflection points of each size ZFC curve. Thus, this is the "good" method for determining the $T_{B}$ distribution, from which it is possible to obtain any of the usual quantities that characterize the distribution like the mean, the mode, or the media. Additionally, it came clear from the results that the MAX and the IP of the polysize ZFC curve are affected not only by the mean size of the particles but also by the size dispersion, and do not coincide neither with the mean, the mode, or the media. Thus, neither IP nor MAX are direct estimators of a characteristic $T_{B}$. This is an interesting result since these values are commonly used in magnetic characterizations and can lead to estimate $T_{B}$ values far from the mean. As an example, for a sample with sigma $=0.5$ and a heating rate of $4 \mathrm{~K} / \mathrm{min}, \mathrm{IP}=0.7\left\langle T_{B}\right\rangle$ and $\operatorname{MAX}=2.12\left\langle T_{B}\right\rangle$. Nevertheless, it was found that the $\mathrm{IP} /\left\langle T_{B}\right\rangle$ ratio depends exclusively on $\sigma$, while $\mathrm{MAX} / T_{B}$ depends also on $K$ and the heating-cooling rate. Therefore, IP cannot be considered as a direct method for obtaining a representative $T_{B}$ value but an "ugly" one, since it differs from $\left\langle T_{B}\right\rangle$ as much as the sample presents size dispersion. This behavior reveals a connection between the IP, one of the most commonly used $T_{B}$ criteria, and the actual mean value of the blocking temperature. For a sample with known $\sigma$, the mean blocking temperature could be obtained from the universal curve presented in this work. This approach of obtaining size distribution information from a universal curve was presented before by Hansen and Mørup ${ }^{26}$ using a rougher model. Regarding MAX, it represents a "bad" estimator of $T_{B}$ due to its dependence on sample and measurement parameters, and its use should be avoided.

In the present development status, the ZFC-FC simulation algorithm does not include a "measurement time" parameter. Just the heating rate is used, so the authors assume that the simulated data points represent the values for "instantaneous" measurements. Therefore, the magnetization values obtained from the simulation would not be equivalent to the ones obtained in an experiment with the same parameters.

Micha's method was applied to characterize a sample of magnetite nanoparticles coated with oleic acid and suspended in hexane. The volume distribution of the sample was obtained from TEM analysis showing a narrow log-normal shape with a mean diameter of $9.5 \mathrm{~nm}$ and a standard deviation of $1.7 \mathrm{~nm}$. In order to obtain an ordered system, the ferrofluid was frozen under a $7 \mathrm{~T}$ magnetic field. Then, a ZFC-FC routine was carried out and a $T_{B}$ distribution was obtained from the data. This distribution was fitted with a $x \operatorname{LnN}[x]$ function using the scale parameter sigma obtained from the TEM data as a fixed fitting parameter. The high goodness of the fitting supports the validity of Micha's method and the low influence of magnetic interaction between particles which is consistent with the particle to particle distance imposed by the FF concentration. Additionally, the resultant IP $/\left\langle T_{B}\right\rangle$ values are consistent with the universal curve obtained from the simulations.

Finally, the effective anisotropy constant of the particles was estimated as the value which gives the maximum coincidence between the ZFC-FC $T_{B}$ distribution and the one obtained from the TEM volume.

The results obtained in this work constitute just a first example of the potential of the presented model in combination with experimental characterization. There is work in progress to enhance the simulation algorithm in order to include the measurement time as a parameter and to consider both inversions processes in the blocking condition.

\section{ACKNOWLEDGMENTS}

The authors would like to acknowledge CONICET and UNLP of Argentina for financial support through Grant Nos. PIP 0720 and X11/680.

${ }^{1}$ G. Reiss and A. Hütten, "Magnetic nanoparticles: Applications beyond data storage," Nat. Mater. 4(10), 725-726 (2005).

${ }^{2}$ Q. A. Pankhurst, J. Connolly, S. K. Jones, and J. J. Dobson, “Applications of magnetic nanoparticles in biomedicine," J. Phys. D: Appl. Phys. 36(13), R167 (2003).

${ }^{3}$ Q. A. Pankhurst, N. T. K. Thanh, S. K. Jones, and J Dobson, "Progress in applications of magnetic nanoparticles in biomedicine," J. Phys. D: Appl. Phys. 42(22), 224001 (2009).

${ }^{4}$ D. Luo, K. A. Carter, and J. F. Lovell, "Nanomedical engineering: Shaping future nanomedicines," Wiley Interdiscip. Rev.: Nanomed. Nanobiotechnol. 7(2), 169-188 (2015).

${ }^{5} \mathrm{~S}$. Dutz and R. Hergt, "Magnetic particle hyperthermia promising tumour therapy?," Nanotechnology 25(45), 452001 (2014).

${ }^{6}$ R. E. Rosensweig, "Heating magnetic fluid with alternating magnetic field,” J. Magn. Magn. Mater. 252, 370-374 (2002).

${ }^{7}$ M. Woińska, J. Szczytko, A. Majhofer, J. Gosk, K. Dziatkowski, and A. Twardowski, "Magnetic interactions in an ensemble of cubic nanoparticles: A Monte Carlo study,” Phys. Rev. B 88(14), 144421 (2013).

${ }^{8}$ E. C. Stoner and E. P. Wohlfarth, "A mechanism of magnetic hysteresis in heterogeneous alloys," Philos. Trans. R. Soc. London, Ser. A 240(826), 599-642 (1948).

${ }^{9}$ C. P. Bean and J. D. Livingston, "Superparamagnetism," J. Appl. Phys. 30(4), S120-S129 (1959).

${ }^{10} \mathrm{C}$. Schmitz-Antoniak, "X-ray absorption spectroscopy on magnetic nanoscale systems for modern applications," Rep. Prog. Phys. 78(6), 062501 (2015).

${ }^{11}$ P. de la Presa, Y. Luengo, V. Velasco, M. P. Morales, M. Iglesias, S. Veintemillas-Verdaguer, P. Crespo, and A. Hernando, "Particle interactions in liquid magnetic colloids by zero field cooled measurements: Effects on heating efficiency," J. Phys. Chem. C 119(20), 11022-11030 (2015).

${ }^{12}$ S. Sankar, A. E. Berkowitz, D. Dender, J. A. Borchers, R. W. Erwin, S. R. Kline, and D. J. Smith, "Magnetic correlations in non-percolated $\mathrm{Co}-\mathrm{SiO}_{2}$ granular films," J. Magn. Magn. Mater. 221(1), 1-9 (2000).

${ }^{13} \mathrm{~F}$. Tournus and A. Tamion, "Magnetic susceptibility curves of a nanoparticle assembly II. Simulation and analysis of ZFC/FC curves in the case of a magnetic anisotropy energy distribution,” J. Magn. Magn. Mater. 323(9), 1118-1127 (2011). 
${ }^{14}$ H. Mamiya, I. Nakatani, T. Furubayashi, and M. Ohnuma, "Analyses of superparamagnetism-magnetic properties of isolated iron-nitride nanoparticles,” Trans. Magn. Soc. Jpn. 2(2), 36-48 (2002).

${ }^{15}$ M. Respaud, J. M. Broto, H. Rakoto, A. R. Fert, L. Thomas, B. Barbara, M. Verelst, E. Snoeck, P. Lecante, A. Mosset et al., "Surface effects on the magnetic properties of ultrafine cobalt particles," Phys. Rev. B 57(5), 2925 (1998).

${ }^{16}$ J. S. Micha, B. Dieny, J. R. Régnard, J. F. Jacquot, and J. Sort, "Estimation of the Co nanoparticles size by magnetic measurements in $\mathrm{Co} / \mathrm{SiO}_{2}$ discontinuous multilayers," J. Magn. Magn. Mater. 272, E967-E968 (2004).

${ }^{17}$ H. Mamiya, M. Ohnuma, I. Nakatani, and T. Furubayashi, "Extraction of blocking temperature distribution from zero-field-cooled and field-cooled magnetization curves," IEEE Trans. Magn. 41(10), 3394-3396 (2005).

${ }^{18}$ J. J. Lu, H. L. Huang, and I. Klik, "Field orientations and sweep rate effects on magnetic switching of Stoner-Wohlfarth particles," J. Appl. Phys. 76(3), 1726-1732 (1994).

${ }^{19}$ N. A. Usov and Y. B. Grebenshchikov, "Hysteresis loops of an assembly of superparamagnetic nanoparticles with uniaxial anisotropy," J. Appl. Phys. 106(2), 023917 (2009).
${ }^{20}$ J. Carrey, B. Mehdaoui, and M. Respaud, "Simple models for dynamic hysteresis loop calculations of magnetic single-domain nanoparticles: Application to magnetic hyperthermia optimization," J. Appl. Phys. 109(8), 083921 (2011).

${ }^{21}$ N. A. Usov, "Numerical simulation of field-cooled and zero field-cooled processes for assembly of superparamagnetic nanoparticles with uniaxial anisotropy," J. Appl. Phys. 109(2), 023913 (2011).

${ }^{22}$ R. Balian and D. Haar, From Microphysics to Macrophysics: Methods and Applications of Statistical Physics (Springer Science \& Business Media, 2006), Vol. 2.

${ }^{23} \mathrm{~L}$. Neel, "Influence des fluctuations thermiques a 1 aimantation des particules ferromagnetiques," C. R. Acad. Sci. 228, 664-668 (1949).

${ }^{24} \mathrm{~A}$. Aharoni, Introduction to the Theory of Ferromagnetism (Oxford University Press, 2000), Vol. 109.

${ }^{25}$ L. F. Shampine and M. W. Reichelt, "The MATLAB ODE suite," SIAM J. Sci. Comput. 18(1), 1-22 (1997).

${ }^{26} \mathrm{M}$. F. Hansen and S. Mørup, "Estimation of blocking temperatures from ZFC/FC curves,” J. Magn. Magn. Mater. 203(1), 214-216 (1999). 